\title{
The epidemiology of symptomatic midfoot osteoarthritis in community-dwelling older adults: cross-sectional findings from the Clinical Assessment Study of the Foot
}

\author{
Martin J. Thomas ${ }^{1 *}$, George Peat ${ }^{1}$, Trishna Rathod ${ }^{1}$, Michelle Marshall ${ }^{1}$, Andrew Moore ${ }^{2}$, Hylton B. Menz ${ }^{1,3}$
} and Edward Roddy ${ }^{1}$

\begin{abstract}
Introduction: The foot is largely overlooked in calls for better characterisation of clinical phenotypes in osteoarthritis $(\mathrm{OA})$. Yet the midfoot complex in particular has the potential to provide important insights into OA pathogenesis given its central role in lower limb load transmission and alignment. Its recent inclusion in radiographic atlases has paved the way for international studies. In this UK study, we provide the first comprehensive account of the descriptive epidemiology of symptomatic midfoot OA.
\end{abstract}

Methods: Participants aged $\geq 50$ years registered with four general practices were recruited via a mailed health survey ( $n=5109$ responders) and research clinic $(n=560$ responders). Symptomatic midfoot OA was defined as midfoot pain in the last 4 weeks, combined with radiographic OA in one or more joints (1st and 2nd cuneometatarsal, navicular first cuneiform and talonavicular joints) graded from weight-bearing dorso-plantar and lateral radiographs using a validated atlas. Prevalence estimates, overall and stratified by age, gender, and socio-economic class, were derived using multiple imputation and weighted logistic regression. Associations between symptomatic midfoot OA and current body mass index, previous injury, history of high-heeled footwear, nodal interphalangeal joint OA and patterns of comorbidity were estimated using binary logistic regression. Healthcare use was summarised.

Results: Symptomatic midfoot OA was present in $12.0 \%$ (95 \% Cl: 10.9, 13.2) of the population aged over 50 years. Higher occurrence was observed in females, adults aged over 75 years, and those in intermediate/routine occupational classes. Obesity, previous foot/ankle injury, and pain in other weight-loaded joints, but not high-heeled footwear or nodal interphalangeal joint $O A$, were associated with increased risk of symptomatic midfoot OA. Persons with symptomatic midfoot OA were also more likely to report multiple non-musculoskeletal comorbidities, including diabetes. In the previous 12 months, the proportions consulting a general practitioner, physiotherapist or podiatrist/chiropodist about foot pain were $46.2 \%, 18.5 \%$ and $47.9 \%$ respectively. A total of $64.7 \%$ had used oral analgesia in the past month for foot pain (36.1\% paracetamol, $31.9 \%$ mild/moderate opioids, $27.7 \%$ NSAIDs).

Conclusions: Our study confirms that symptomatic OA frequently affects the midfoot. The patterns of associations are interpreted as being largely consistent with the role of mechanical factors in its pathogenesis.

\footnotetext{
* Correspondence: m.thomas@keele.ac.uk

${ }^{1}$ Arthritis Research UK Primary Care Centre, Research Institute for Primary Care and Health Sciences, Keele University, Keele, Staffordshire, ST5 5BG, UK Full list of author information is available at the end of the article
} 


\section{Introduction}

Symptomatic osteoarthritis (OA) is now widely accepted as being a multifactorial pathology affecting the whole joint complex [1]. The different aetiology, risk factors and prognosis seen across different joint sites, such as the hip, knee and hand, suggest that characterisation of discrete clinically relevant OA phenotypes is necessary to enable targeted treatment interventions to be developed [2].

Although the foot has largely been neglected relative to other sites commonly affected by OA [3], persistent foot pain is common in older adults [4], with approximately one in six estimated to have symptomatic foot OA [5]. As with other regions of the body, OA of the foot joint complex may comprise more than one phenotype. Recent latent class analysis of radiographic foot OA at five key sites (1st metatarsophalangeal joint (MTPJ), 1st and 2nd cuneometatarsal joint (CMJs), navicular first cuneiform joint (NCJ) and talonavicular joint (TNJ)) identified an isolated bilateral 1st MTPJ OA group and a polyarticular midfoot-dominant OA group as possible distinct radiographic phenotypes [6]. In the foot, the predominant focus of epidemiological studies has been on OA at the 1st MTPJ [3]. However, better characterisation of a symptomatic midfoot OA phenotype could facilitate more targeted clinical assessment and management, particularly as primary care diagnosis of foot symptoms is often limited and unclear $[7,8]$.

The midfoot also presents a particularly interesting complex in which to investigate OA given its important load distribution function [9], enabling the foot to conform to terrain and yet provide a rigid lever for forward motion when walking. It remains highly speculative whether OA in the midfoot has important implications for the development of $\mathrm{OA}$ in adjacent joints. Case series reporting high rates of $\mathrm{OA}$ in adjacent joints following midfoot fusion (for example, $[10,11]$ ) have lacked appropriate control observations and may be confounded by factors associated with both midfoot fusion and $\mathrm{OA}$ in other foot joints.

The objectives of this study were to: (i) provide population prevalence estimates for symptomatic midfoot OA (including estimates for midfoot pain and disabling symptomatic midfoot $\mathrm{OA}$ ) in adults aged 50 years and over, (ii) examine the association with selected potential aetiological factors, (iii) describe associated patterns of comorbidity, and (iv) determine the frequency of selected healthcare use for foot pain among persons with symptomatic midfoot $\mathrm{OA}$.

\section{Methods}

Study design

This paper utilises baseline data from a populationbased prospective observational cohort study, the Clinical
Assessment Study of the Foot (CASF) [12]. Adults aged 50 years and over registered with four general practices in North Staffordshire, United Kingdom, were invited to take part in the study, irrespective of foot-related consultation. Ethical approval was obtained from Coventry Research Ethics Committee (REC reference number: 10/ H1210/5) and all participants gave their written consent to participate.

\section{Data collection}

At baseline, eligible participants were mailed a health survey that included general health (Short Form-12 (SF12)) [13], Hospital Anxiety and Depression Scale (HADS) [14], comorbidities, anthropometric characteristics (selfreported height and weight), foot pain, footwear, healthcare consultation, pain medication use, and demographic and socio-economic characteristics (age, gender, marital status, education, current employment status, and occupation). Foot pain questions included: pain in the foot in the last 12 months; pain, aching or stiffness in the foot in the last month [15], number of days with foot pain in the last 12 months; and the Manchester Foot Pain and Disability Index (MFPDI) [16]. The location of foot pain in the last 4 weeks was ascertained from shading a foot manikin (๔ The University of Manchester 2000. All rights reserved) [17]. Participants who reported foot pain in the last 12 months and provided written consent to further contact were invited to attend a research assessment clinic where weight-bearing dorso-plantar and lateral radiographs of each foot, clinical interview and physical examination were undertaken, in accordance with defined standardised protocols $[12,18]$. Health survey responders were also invited to consent to medical record review.

\section{Scoring of radiographs and case definitions}

Plain radiographs were scored by a single reader (MM) blinded to all other participant information. Osteophytes and joint space narrowing at the 1st and 2nd CMJs, NCJ and TNJ were scored (0-3) according to a validated atlas and classification system [18]. Eight weeks later a random selection of radiographs from 60 participants were re-scored by $\mathrm{MM}$ and independently by HBM. Intrarater and inter-rater reliability for the presence of $\mathrm{OA}$ in a midfoot joint were excellent (mean $\mathrm{k}=0.95$, mean \% agreement $=99 \%)$ and moderate $($ mean $\mathrm{\kappa}=0.40$, mean $\%$ agreement $=78 \%$ ) respectively.

Midfoot pain was defined as self-reported pain in the last 4 weeks by shading the midfoot region on a foot manikin, designated using a pre-defined regional marking template $[17,19]$.

Symptomatic midfoot OA was defined as a radiographic score of 2 or more for osteophytes or joint space narrowing on either weight-bearing dorso-plantar or lateral views, in one or more midfoot joints (1st CM), 2nd 
CMJ, NCJ or TNJ), and midfoot pain in the last 4 weeks in the same foot (as defined above).

Disabling symptomatic midfoot OA was defined as symptomatic radiographic OA together with at least one of the ten items within the MFPDI function construct scored at the level of 'on most/every day(s)' [20]. If all items were scored at the level of 'none of the time' or 'on some days', symptomatic midfoot OA was classed as nondisabling. Individuals were defined as having any of the above case definitions if either or both feet were affected.

Individuals identified as having non-specific inflammatory arthritis, rheumatoid arthritis, or psoriatic arthritis were excluded from the analyses based on medical record review (primary care and local hospital) or clinical X-ray report by a consultant musculoskeletal radiologist [5].

\section{Statistical analysis}

\section{Estimating population prevalence}

Using baseline health survey and radiographic data, the population prevalence of midfoot pain, symptomatic midfoot $\mathrm{OA}$ and disabling symptomatic midfoot $\mathrm{OA}$ in the individual were estimated using multiple imputation and weighted logistic regression modelling. Multiple imputation was used to account for missing item-level data from the health survey and estimates were then weighted to take into account selective non-response to the health survey [21]. Missing data were inspected to ensure that the missing at random assumption was reasonable.

Imputation involved all baseline responders and utilised the following variables: age, gender, general practice, social class, marital status, number of days with foot pain in the last 12 months, Rasch-transformed MFPDI interval level scores for the pain and function constructs [22], individual MFPDI function items to estimate disabling symptoms [20], self-reported foot pain, aching, or stiffness in the last month, SF-12 score, HADS score, and radiographic foot $\mathrm{OA}$ and pain regions. Fifteen imputed datasets were generated and combined using Rubin's combination rules [23]. Prevalence estimates (and $95 \%$ confidence intervals $(\mathrm{CI})$ ) were calculated using the mim: proportion command and applied to the total baseline responder population. Selective non-response to the health survey was handled by generating weighted estimates that likely reflect the profile of non-responders using information available for the whole eligible baseline population (age, gender and general practice). Weighted logistic regression was combined with the imputed datasets to generate prevalence estimates (and $95 \% \mathrm{CI}$ ) in the whole eligible baseline population. Crude population prevalence estimates for midfoot pain, symptomatic radiographic midfoot $\mathrm{OA}$ and disabling radiographic symptomatic midfoot $\mathrm{OA}$ were then stratified by gender, age, and socio-economic class, based on occupation. This approach to estimating population prevalence mirrors our procedures adopted for estimating overall symptomatic radiographic foot OA [5].

The analyses described below were conducted using data from the CASF clinic cohort. A complete case approach was taken due to the generally very low levels of missing data within clinic participants.

\section{Potential aetiological determinants of interest}

Binary logistic regression estimated the crude, and as appropriate, age-gender-body mass index (BMI)-adjusted associations between symptomatic midfoot $\mathrm{OA}$ and selected variables. Obesity $\left(\geq 30 \mathrm{~kg} / \mathrm{m}^{2}\right)$ at time of baseline assessment was calculated from clinic-measured height and weight. Lifetime recall of previous foot and ankle injury was ascertained on standardised personal interview (yes/no response to the question 'Have you ever injured your feet or ankles?', with follow-up questions to ascertain type, anatomical location and duration of time since injury, analysed in right feet only). Lifetime recall of frequent use of high-heeled footwear among females was ascertained from a health survey item on footwear [12] (high frequency use was defined as reported use of highheeled shoes on most days for at least one 10-year period between the age of 20 and 49 years). Nodal interphalangeal joint (IPJ) OA was defined as a Kellgren and Lawrence [24] score of two or more, in two or more IPJs (digits 2-5) and the presence of two or more Heberden or Bouchard nodes (digits 2-5) across either hand [25].

\section{Associated impairment and comorbidities}

The following self-reported impairment and comorbidities were ascertained from the health survey and their associations with symptomatic midfoot $\mathrm{OA}$ examined using binary logistic regression: general health and function (SF-12 physical and mental components, with each variable dichotomised around the median of the data distribution), HADS score (categorised as normal, mild, moderate or severe), chest problems, heart problems, deafness, eyesight problems, hypertension, diabetes, stroke, cancer, circulation problems in the legs, intermittent claudication (defined by the Edinburgh Claudication Questionnaire [26]) and co-occurring joint pain in the last month at other weight-loaded sites (low back, hip, knee, hindfoot/ankle and forefoot). Pain location was defined using recognised body and foot manikins and templates (low back [27]; hip [28]; knee [29]; foot and ankle [17]) that have demonstrated excellent inter- and intra-rater reliability [19, 29]. Crude odds ratios (OR) with $95 \%$ CI were reported together with estimates adjusted for age and gender.

\section{Frequency of primary healthcare use}

Among adults with symptomatic midfoot OA, the frequency of foot pain-related consultation with a general 
practitioner (GP) or allied health professional (physiotherapist or podiatrist/chiropodist) was summarised as the 12-month period prevalence, and the proportion of consultations that were with the National Health Service (NHS) or private practice was described. The frequency of medication use for foot pain among adults with symptomatic midfoot OA was summarised as 1-month period prevalence.

All analyses were conducted using STATA V.12.0 (StataCorp, College Station, TX, USA).

\section{Results}

The description of participants recruited into the study has been previously reported [5]. Briefly, in 2010/2011, 9403 potentially eligible adults were identified. Following initial screening, 9194 were posted a health survey, with 5109 responding (adjusted response $56 \%$ ). Following further screening, 1634 eligible participants were invited to a research clinic, 560 (34 \%) of whom attended. In total, 525 contributed to the final analyses following the exclusion of those with incomplete foot pain data $(n=8)$, incomplete radiographic data $(\mathrm{n}=3)$ and inflammatory arthritis $(\mathrm{n}=24)$.

\section{Population prevalence}

The population prevalence of midfoot pain in the last month among adults aged 50 years and over was $19.4 \%$ (95\% CI: $18.3 \%$, $20.5 \%$ ). The corresponding estimate for symptomatic midfoot OA was $12.0 \%$ (95 \% CI: $10.9 \%$, $13.2 \%)$ and for disabling symptomatic midfoot OA was $9.6 \%$ (95 \% CI: $8.6 \%, 10.6 \%$ ). Based on the imputed estimates, a prevalence staircase was constructed for a denominator population of 10, 000 persons aged 50 years and over (Fig. 1). Prevalence was higher in females, increased most notably in females aged 75 years and over, and was inversely related to socio-economic class (Table 1).

Among clinic attenders, 149 individual feet were diagnosed with symptomatic midfoot OA. Of these, most feet $(\mathrm{n}=100,67 \%)$ had only one joint involved, with the 2nd CMJ being most commonly affected ( $\mathrm{n}=39,39 \%)$, followed by the TNJ $(n=36,36 \%), 1$ st CMJ $(n=13$, $13 \%)$ then $\mathrm{NCJ}(\mathrm{n}=12,12 \%)$.

\section{Potential aetiological determinants of interest}

Symptomatic midfoot OA was positively associated with BMI. However, for self-reported previous foot or ankle injury, previous frequent use of high-heeled footwear among females and nodal IPJ OA, the $95 \%$ CI for these estimates crossed unity (Table 2). Further analyses of previous foot or ankle injury among right feet suggested that those with symptomatic midfoot OA were more likely to recall having a fracture (Table 3). Examining all foot and ankle injuries to the right foot $(n=42$, with 49 injuries), the ankle was reported as the most commonly affected location, particularly for a sprain (41\%), and this was followed by forefoot fracture (16\%) and other forefoot injuries (14\%). Additionally, most recalled injuries occurred over 10 years prior to baseline clinic assessment (56 \%) (Table S1 in Additional file 1).

\section{Associated impairment and comorbidities}

Strong positive associations were observed between symptomatic midfoot $\mathrm{OA}$ and impaired physical function measured by the SF-12, mild and moderate anxiety and mild depression measured using the HADS scores, and diabetes. Following further adjustment for BMI, the positive association with diabetes was attenuated (OR, 1.48; 95 \%CI: 0.85, 2.55). Observed associations between symptomatic midfoot $\mathrm{OA}$ and four or more comorbidities, and self-reported pain at all weight-loaded joint sites of the body were positive. For co-occurring joint pain, increased magnitude but reduced precision was observed for sites closest to the midfoot (hindfoot/ankle and forefoot) (Table 4). The observed positive associations across all the weight-loaded joint sites remained following further adjustment for BMI (data not shown).

\section{Frequency of primary healthcare use}

Most participants with symptomatic midfoot OA consulted a healthcare professional in the last 12 months for foot pain, with the frequency of GP and podiatrist/ chiropodist consultations being similar (Table 5). Among individuals with symptomatic midfoot $\mathrm{OA}$ in either foot ( $\mathrm{n}=119), 16 \%$ had accessed private allied health professional healthcare for foot pain in the last 12 months

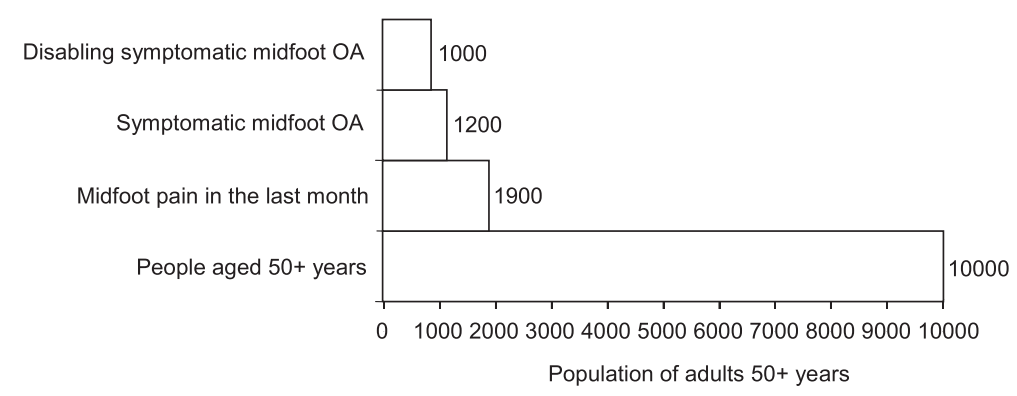

Fig. 1 Prevalence staircase for symptomatic midfoot OA 
Table 1 Population prevalence of midfoot pain, symptomatic, and disabling symptomatic midfoot OA by demographic characteristics

\begin{tabular}{|c|c|c|c|}
\hline & Midfoot pain & Symptomatic midfoot OA & Disabling symptomatic midfoot OA \\
\hline All adults aged $50+$ & $19.4(18.3,20.5)$ & $12.0(10.9,13.2)$ & $9.6(8.6,10.6)$ \\
\hline \multicolumn{4}{|l|}{ Gender } \\
\hline Male & $17.2(15.7,18.7)$ & $10.3(8.9,11.7)$ & $7.9(6.7,9.1)$ \\
\hline Female & $21.5(19.9,23.1)$ & $13.7(12.0,15.5)$ & $11.2(9.7,12.8)$ \\
\hline \multicolumn{4}{|l|}{ Age (years) overall } \\
\hline $50-64$ & $20.6(19.0,22.2)$ & $11.8(10.0,13.5)$ & $9.0(7.5,10.4)$ \\
\hline $65-74$ & $17.6(15.7,19.5)$ & $11.1(9.4,12.8)$ & $8.8(7.2,10.4)$ \\
\hline $75+$ & $18.7(16.3,21.1)$ & $14.4(11.9,16.9)$ & $13.0(10.7,15.3)$ \\
\hline \multicolumn{4}{|l|}{ Males } \\
\hline $50-64$ & $18.8(16.6,21.0)$ & $10.5(8.4,12.6)$ & $7.5(5.9,9.1)$ \\
\hline $65-74$ & $15.1(12.5,17.6)$ & $9.3(6.9,11.7)$ & $7.8(5.5,10.0)$ \\
\hline $75+$ & $15.4(11.8,18.9)$ & $11.3(8.0,14.6)$ & $9.9(6.8,12.3)$ \\
\hline \multicolumn{4}{|l|}{ Females } \\
\hline $50-64$ & $22.4(20.1,24.7)$ & $13.1(10.6,15.5)$ & $10.5(8.3,12.6)$ \\
\hline $65-74$ & $20.2(17.3,23.1)$ & $12.9(10.1,15.8)$ & $9.9(7.4,12.4)$ \\
\hline $75+$ & $21.1(17.8,24.4)$ & $16.6(13.2,20.0)$ & $15.3(12.1,18.5)$ \\
\hline \multicolumn{4}{|l|}{ Socio-economic classification } \\
\hline Managerial and professional & $12.7(10.6,14.7)$ & $6.9(4.9,8.9)$ & $4.9(3.3,6.5)$ \\
\hline Intermediate occupations & $18.8(16.2,21.4)$ & $12.6(9.9,15.4)$ & $10.5(8.2,12.8)$ \\
\hline Routine and manual & $21.6(20.0,23.2)$ & $13.3(11.6,15.0)$ & $10.5(9.1,11.9)$ \\
\hline
\end{tabular}

Based on imputed and weighted analyses

$O A$ osteoarthritis

(22\%, n $=5$ of physiotherapy consultations, and $25 \%$, $\mathrm{n}=14$ of podiatry/chiropody consultations).

One-month period prevalence of pain medication use for foot pain was $70.6 \%$ (95 \% CI: 62.3, 78.9) (Table 5). One-month period prevalence for one or more form of oral pain medication use (paracetamol, non-steroidal antiinflammatory drugs, including coxibs and mild/moderate opioids) was 64.7 \% (95 \% CI: 55.9, 73.4). Pain medication and topical applications were more frequently used than herbal/nutraceutical preparations.

\section{Discussion}

This is the first comprehensive account describing the epidemiology of symptomatic midfoot OA. Our findings suggest that symptomatic midfoot OA is common, being present in an estimated $12 \%$ of the population aged 50 years and over. The higher occurrence of symptomatic midfoot $\mathrm{OA}$ in females, older age, and lower socio-economic classes is consistent with previous epidemiological studies of foot pain and other sites of musculoskeletal pain [4, 30].

The associations with obesity, previous injury and pain at all other weight-loaded joint sites but not nodal IPJ OA (a proxy for more widespread OA [31]), together with the load distribution function of the midfoot [9], are consistent with the role of mechanical factors in its pathogenesis. Whilst no one underlying mechanism (e.g. inflammatory or mechanical) appears to be responsible in its entirety for the development of OA [32, 33], the characteristics of deterioration associated with structural and pathological changes suggest that altered biomechanics and joint loading are important modifiable mediators of onset and progression, particularly in the lower limb [34]. At the knee, epidemiological studies have shown malalignment and aberrant loading to be associated with OA development (for example, [35, 36]). As a functional unit, the midfoot is highly sensitive to aberrant biomechanics and altered joint loading due to its load distribution function when walking $[37,38]$, and our epidemiological findings provide additional evidence in support of a malalignment loading hypothesis. Within the midfoot joints imaged and scored, isolated midfoot joint involvement was the predominant observation, with the 2nd CMJ being the most frequently affected joint, followed by the TNJ. The mechanical vulnerability of the 2nd CMJ as the more rigid apex of the transverse arch [39] appears consistent with a greater susceptibility to aberrant loading compared with the TNJ, which structurally has more 
Table 2 Relationship between selected potential aetiological determinants of interest and symptomatic midfoot OA

\begin{tabular}{|c|c|c|c|c|}
\hline & Total N & SMOA N & Crude OR (95 \% Cl) & Adjusted OR (95\% Cl) \\
\hline \multicolumn{5}{|c|}{ Body mass index $\left(\mathrm{kg} / \mathrm{m}^{2}\right)$} \\
\hline Non-obese $<30$ & 286 & 49 & 1 & 1 \\
\hline Obese $\geq 30$ & 235 & 69 & $2.01(1.33,3.05)$ & $2.02(1.32,3.08)^{\mathrm{a}}$ \\
\hline \multicolumn{5}{|c|}{ Self-reported previous injury to either foot or ankle } \\
\hline No & 166 & 29 & 1 & 1 \\
\hline Yes & 359 & 90 & $1.58(0.99,2.52)$ & $1.60(0.98,2.60)^{b}$ \\
\hline \multicolumn{5}{|c|}{ Self-reported frequency of use of high-heeled footwear ${ }^{c}$} \\
\hline Low & 78 & 18 & 1 & 1 \\
\hline High & 211 & 47 & $0.96(0.51,1.77)$ & $0.98(0.51,1.88)^{d}$ \\
\hline \multicolumn{5}{|l|}{ Nodal IPJ OA ${ }^{e}$} \\
\hline No & 402 & 85 & 1 & 1 \\
\hline Yes & 123 & 34 & $1.42(0.90,2.26)$ & $1.32(0.80,2.16)^{b}$ \\
\hline
\end{tabular}

Based on complete case clinic data

$O A$ osteoarthritis, SMOA symptomatic midfoot osteoarthritis, OR odds ratio, Cl confidence interval, IPJ interphalangeal joint

${ }^{a}$ Estimate adjusted for age and gender

${ }^{b}$ Estimate adjusted for age, gender and body mass index

'Examination was restricted to females and the exposure was defined as previous footwear (low-versus high-heeled shoes) worn on most days for at least one

10 -year period between 20 and 49 years old

${ }^{\mathrm{d}}$ Estimate adjusted for age and body mass index

${ }^{\mathrm{e}}$ Nodal interphalangeal joint (IPJ) OA defined as Kellgren and Lawrence $\geq 2$ in $\geq 2$ IPJs (digits 2-5), and the presence of $\geq 2$ Heberden or Bouchard nodes

(digits 2-5) across either hand [25]

functional capacity to accommodate larger gravitational loading effects, together with shear forces during both static and dynamic functional tasks. Different patterns of single joint involvement may reflect different midfoot loading patterns. Although the observed positive associations between symptomatic midfoot OA and obesity extend earlier work, showing that being obese is associated with non-specific foot pain [40], further research is required to confirm or refute a more systemic counterargument for the involvement of obesity [41].
The observed trend that previous injury is associated with symptomatic midfoot OA supports expert clinical opinion that OA changes in the midfoot commonly follow trauma [42] and mirrors a well-established association between injury and OA in the knee [43]. However, more research is needed to better understand the relationships between symptomatic midfoot $\mathrm{OA}$ and transient minor traumas versus chronic injury mechanisms and consequences. Whilst footwear selection for females may also precipitate a range of foot-related problems

Table 3 Associations between right symptomatic midfoot $O A$ and previous right foot or ankle injury

\begin{tabular}{|c|c|c|c|c|}
\hline Self-reported right foot or ankle injury & Total N & RSMOA N & Crude OR (95\% Cl) & Adjusted OR $(95 \% \mathrm{Cl})^{\mathrm{a}}$ \\
\hline \multicolumn{5}{|l|}{ One or more previous injury } \\
\hline No & 269 & 37 & 1 & 1 \\
\hline Yes & 256 & 42 & $1.23(0.76,1.99)$ & $1.30(0.79,2.12)$ \\
\hline \multicolumn{5}{|l|}{ One or more previous sprain } \\
\hline No & 342 & 56 & 1 & 1 \\
\hline Yes & 167 & 21 & $0.73(0.43,1.26)$ & $0.80(0.46,1.39)$ \\
\hline \multicolumn{5}{|l|}{ One or more previous fracture } \\
\hline No & 433 & 59 & 1 & 1 \\
\hline Yes & 83 & 20 & $2.01(1.13,3.57)$ & $2.06(1.14,3.71)$ \\
\hline \multicolumn{5}{|l|}{ One or more previous other injury } \\
\hline No & 443 & 65 & 1 & 1 \\
\hline Yes & 72 & 12 & $1.16(0.59,2.28)$ & $1.08(0.54,2.15)$ \\
\hline
\end{tabular}

Based on complete case clinic data

$O A$ osteoarthritis, $R S M O A$ right symptomatic midfoot osteoarthritis, $O R$ odds ratio, $\mathrm{Cl}$ confidence interval

aEstimates adjusted for age, gender and body mass index 
Table 4 Associated self-reported impairment and comorbidities among adults with symptomatic midfoot OA

SF-12 Physical impairment ${ }^{\mathrm{b}}$

No

Yes

SF-12 Mental impairment ${ }^{\text {b }}$

No

Yes

HADS anxiety

Normal (0-7)

Mild (8-10)

Moderate (11-14)

Severe (15-21)

HADS depression

Normal (0-7)

Mild (8-10)

Moderate (11-14)

Severe (15-21)

Chest problems

No

Yes

Heart problems

No

Yes

Deafness

$$
\text { No }
$$

Yes

Eyesight problems ${ }^{c}$

$$
\text { No }
$$

Yes

Hypertension

$$
\begin{aligned}
& \text { No } \\
& \text { Yes }
\end{aligned}
$$

Diabetes

No

Yes

Stroke

No

Yes

Cancer

$$
\text { No }
$$

Yes

Circulation problems in legs

Yes
Total N

242

245

240

247

289

114

92

23

371

86

50

11

411

114

424

101

401

124

381

144

284

241

444

81

492

33

493

32

358

167
SMOA N

30

76

45

61

52

30

29

6

71

27

16

3

87

32

88

31

89

30

82

37

53

66

90

29

113

6

109

10

68

51
Crude OR (95\% Cl)

1

$3.18(1.99,5.08)$

1

$1.42(0.92,2.19)$

1

$1.63(0.97,2.72)$

$2.10(1.23,3.57)$

$1.61(0.60,4.28)$

1

$1.93(1.15,3.26)$

$1.99(1.04,3.80)$

$1.58(0.41,6.12)$

1

$1.45(0.91,2.33)$

$1.34(0.83,2.18)$

1

$1.69(1.04,2.74)$

$1.48(0.90,2.43)$

$0.90(0.55,1.49)$

$1.12(0.70,1.80)$

$1.13(0.71,1.78)$

$1.26(0.81,1.97)$

1

$1.40,(0.92,2.15)$

$1.64(1.09,2.48)$

1

$2.19(1.32,3.65)$

1

$1.93(1.14,3.25)$

1

$0.59(0.23,1.49)$

$0.75(0.30,1.85)$

1

$1.19(0.54,2.66)$

$1.60(0.74,3.48)$

1

$1.52(0.97,2.38)$ 
Table 4 Associated self-reported impairment and comorbidities among adults with symptomatic midfoot OA (Continued)

\begin{tabular}{|c|c|c|c|c|}
\hline \multicolumn{5}{|c|}{ Intermittent claudication $^{\mathrm{d}}$} \\
\hline No & 443 & 94 & 1 & 1 \\
\hline Yes & 20 & 4 & $0.93(0.30,2.84)$ & $0.82(0.26,2.56)$ \\
\hline \multicolumn{5}{|c|}{ Non-musculoskeletal comorbidities ${ }^{\mathrm{e}}$} \\
\hline $0-1$ & 167 & 23 & 1 & 1 \\
\hline $2-3$ & 153 & 30 & $1.53(0.84,2.77)$ & $1.31(0.71,2.41)$ \\
\hline $4+$ & 137 & 43 & $2.86(1.62,5.06)$ & $2.23(1.23,4.05)$ \\
\hline \multicolumn{5}{|c|}{ Low back pain } \\
\hline No & 289 & 49 & 1 & 1 \\
\hline Yes & 236 & 70 & $2.07(1.36,3.13)$ & $2.09(1.37,3.19)$ \\
\hline \multicolumn{5}{|c|}{ Hip pain } \\
\hline No & 321 & 49 & 1 & 1 \\
\hline Yes & 204 & 70 & $2.90(1.91,4.41)$ & $3.08(2.00,4.74)$ \\
\hline \multicolumn{5}{|c|}{ Knee pain } \\
\hline No & 293 & 51 & 1 & 1 \\
\hline Yes & 232 & 68 & $1.97(1.30,2.98)$ & $2.12(1.38,3.25)$ \\
\hline \multicolumn{5}{|c|}{ Hindfoot/ankle pain } \\
\hline No & 234 & 27 & 1 & 1 \\
\hline Yes & 291 & 92 & $3.54(2.21,5.68)$ & $3.63(2.25,5.86)$ \\
\hline \multicolumn{5}{|c|}{ Forefoot pain } \\
\hline No & 187 & 18 & 1 & 1 \\
\hline Yes & 338 & 101 & $4.00(2.33,6.86)$ & $4.50(2.59,7.82)$ \\
\hline \multicolumn{5}{|c|}{ Other lower limb pain ${ }^{f}$} \\
\hline No & 77 & 3 & 1 & 1 \\
\hline Yes & 448 & 116 & $8.62(2.67,27.87)$ & $8.53(2.63,27.71)$ \\
\hline
\end{tabular}

Based on complete case clinic data

OA osteoarthritis, SMOA symptomatic midfoot osteoarthritis, OR odds ratio, Cl confidence interval, SF-12 Short Form-12 [13], HADS Hospital Anxiety and Depression Scale [14]

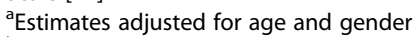

${ }^{b}$ Variable dichotomised around the median of the data distribution

'Excludes the need for glasses

${ }^{\mathrm{d}}$ Defined as calf pain when walking at an ordinary pace on level ground (including uphill or when hurried) that disappears in 10 min or less by standing still [26]

${ }^{e}$ Count based on each of the above self-reported variables, excluding SF-12 scores (HADS scores $\geq 8$ )

fVariable includes presence of pain in hip, knee, hindfoot/ankle or forefoot

secondary to altered biomechanics [44], the consequences of these alterations may be more pronounced in other regions of the foot, such as the forefoot and toes.

The proportion of participants with symptomatic midfoot OA who reported consulting a GP in the last 12-months for foot pain (46\%) was high compared to previous population estimates generated within North Staffordshire for other joint pain and problems among adults aged 50 years and over (self-reported consultation for knee pain, $33 \%$ in the last 12 months [45]; and selfreported hand problems, $22 \%$ in last 12 months [46]). A record-based review of consultation for musculoskeletal foot problems in the last 18 months among adults aged 50 years and over was previously estimated at $12 \%$ [47]. The higher estimate obtained for consultation for symptomatic midfoot OA seen in our study could possibly arise as a result of concurrent pain elsewhere in the foot contributing to consultation, inaccurate recall of the last 12 months, and consultation rates being higher in a subgroup of self-selecting volunteers who attended a foot research clinic and may have more severe problems.

Nearly three-quarters of participants had recently used some form of pain medication for foot pain. Whilst the frequent use of oral and topical medications would appear in keeping with clinical guidelines for OA in general, one-fifth of participants had recently used herbal or nutraceutical preparations, which is inconsistent with current recommendations [48].

Frequent healthcare use and associated disability must be interpreted at the level of the foot given the high proportion of people with symptomatic midfoot OA who have hindfoot/ankle pain and forefoot pain. However, this 
Table 5 Frequency of selected healthcare use for foot pain

\begin{tabular}{lll}
\hline Healthcare use for foot pain & \multicolumn{2}{l}{$\begin{array}{c}\text { Adults with symptomatic midfoo } \\
\text { OA }(n=119)\end{array}$} \\
\cline { 2 - 3 }$N$ & \multicolumn{2}{c}{$\begin{array}{l}\text { Proportion } \\
\%(95 \% \mathrm{Cl})\end{array}$} \\
\hline Healthcare professional consulted in last 12 months \\
GP & 55 & $46.2(37.1,55.3)$ \\
Physiotherapist & 22 & $18.5(11.4,25.6)$ \\
Podiatrist/chiropodist & 57 & $47.9(39.0,57.0)$ \\
Any of the above & 86 & $72.3(64.1,80.4)$ \\
Pain medication in last month & & \\
Paracetamol & 43 & $36.1(27.4,44.9)$ \\
Mild/moderate opioids & 38 & $31.9(23.4,40.4)$ \\
Topical cream/gel/spray & 37 & $31.1(22.7,39.5)$ \\
NSAIDs, including coxibs & 33 & $27.7(19.6,35.9)$ \\
Herbal/nutraceuticals & 25 & $21.0(13.6,28.4)$ \\
Any of the above & 84 & $70.6(62.3,78.9)$
\end{tabular}

Based on complete case clinic data

$O A$ osteoarthritis, $\mathrm{Cl}$ confidence interval, GP general practitioner, NSAIDs non-steroidal anti-inflammatory drugs

study indicates that people with symptomatic midfoot OA may require management and are frequently present in primary care, though often in the context of multimorbidity and not necessarily as a discrete phenotype. The overwhelming majority (97\%) of individuals with symptomatic midfoot OA will report pain at other lower extremity joints and the extent of this comorbidity is greater than expected by chance alone. Shared aetiological pathways would carry implications for clinical care, for example, in addressing common causes of lower extremity pain, in biomechanically based interventions aimed at the lower extremity as a functional unit, and in 'collateral benefits' to lower extremity pain of effective local treatment to one joint. For the local management of symptomatic midfoot $\mathrm{OA}$, there is limited evidence for interventions such as foot orthoses [49], intra-articular corticosteroid/local anaesthetic injections [50] and arthrodesis [51]. Better characterisation of this phenotype may help to inform more effective targeted treatments.

Strengths of this study include census sampling from general practice registers and the use of multiple imputation and weighted logistic regression to account for selection bias. Although our approach adopts recognised statistical adjustment techniques [21], these estimates should be viewed cautiously due to non-response. Whilst causality cannot be inferred for obesity, OA at the knee has been shown to cumulate through adulthood with increased exposure to elevated BMI [52], and reverse association (symptomatic midfoot $\mathrm{OA}$ causing weight gain) seems less plausible. The majority of self-reported injuries occurred over 10 years ago and high-heeled footwear exposure (between 20 and 49 years old) predated the sample age ( $\geq 50$ years old), suggesting that these exposures appear to predominantly occur earlier in life.

The study has some noteworthy limitations. The clinical sample all had foot pain in the last 12 months. Therefore, prevalence estimates represent symptomatic individuals and all associations are relative to foot pain elsewhere. Consequently, observed associations may underestimate the true effect. Despite excellent intra-rater reliability for scoring the presence of $\mathrm{OA}$ in a joint, inter-rater reliability was moderate. Furthermore, we noted that the main scorer MM was systematically more conservative compared to HBM. Therefore, the reported population prevalence estimates may be underestimated. Also, estimates for previous injury, previous footwear, comorbidity and healthcare use were derived from self-report data, and laterality for co-occurring joint pain was not considered. The footwear question designed specifically for this study has not been validated. Both footwear and previous injury may be particularly vulnerable to recall bias, however, attempts were made to minimise these issues. First, in the standardised clinical interview all participants were probed in relation to a variety of previous injury exposures over a number of questions to promote and assist recall. Second, previous footwear was asked of all participants in the health survey irrespective of foot pain status. Finally, the selected healthcare use estimates are based on small numbers and therefore could only be estimated imprecisely.

\section{Conclusions}

Symptomatic midfoot OA occurs commonly in the population aged 50 years and over. Its relationship with demographic and socio-economic factors is similar to other forms of OA but the pattern of association with potential determinants, together with the form and function of the midfoot, suggest a central role for mechanical load in this phenotype. As such it may provide a useful model for further studies of mechanical load in OA pathogenesis. Clinical research could focus on the effectiveness of existing and emerging non-pharmacological treatments for this phenotype.

\section{Additional file}

Additional file 1: Table S1. Foot or ankle injury location and duration of time since injury among adults with symptomatic midfoot $\mathrm{OA}$ in the right foot.

\section{Abbreviations}

BMI: body mass index; CASF: Clinical Assessment Study of the Foot; $\mathrm{Cl}$ : confidence interval; CMJ: cuneometatarsal joint; GP: general practitioner; HADS: Hospital Anxiety and Depression Scale; IPJ: interphalangeal joint; MFPDI: Manchester Foot Pain and Disability Index; MTPJ: metatarsophalangeal joint; NCJ: navicular first cuneiform joint; NHS: National Health Service; NSAIDs: Non-steroidal anti-inflammatory drugs; OA: osteoarthritis; OR: odds ratio; SF-12: Short Form-12; TNJ: talonavicular joint. 


\section{Competing interests}

The authors declare that they have no competing interests.

\section{Authors' contributions}

MJT, ER, GP, AM and HBM conceived the study. MJT, ER, GP and AM designed the study. MJT, ER and MM were responsible for data acquisition. Analysis was undertaken by MJT and TR. All authors interpreted data, drafted or revised the manuscript critically for important intellectual content, and approved the final version of the manuscript.

\section{Acknowledgements}

We would like to thank the administrative, health informatics and research nurse teams of Keele University's Arthritis Research UK Primary Care Centre, the staff of the participating general practices and the Haywood Hospital, particularly Dr Jackie Saklatvala, Carole Jackson and the radiographers at the Department of Radiology. We would like to acknowledge the contributions of Linda Hargreaves, Gillian Levey, Liz Mason, Dr Jennifer Pearson, Julie Taylor and Dr Laurence Wood to data collection. We would also like to thank Adam Garrow and the University of Manchester for permission to use the foot manikin (๔ The University of Manchester 2000. All rights reserved).

\section{Funding}

This work was funded by an Arthritis Research UK Programme Grant (18174) and service support through West Midlands North CLRN. The study funders had no role in the study design; data collection, analysis or interpretation; in the writing of the manuscript; or in the decision to submit the manuscript for publication. MJT was supported by West Midlands Strategic Health Authority through a Nursing, Midwifery, and Allied Health Professionals Doctoral Research Training Fellowship (NMAHP/RTF/10/02). HBM is currently a National Health and Medical Research Council of Australia Senior Research Fellow (ID: 1020925).

\section{Author details}

${ }^{1}$ Arthritis Research UK Primary Care Centre, Research Institute for Primary Care and Health Sciences, Keele University, Keele, Staffordshire, ST5 5BG, UK. ${ }^{2}$ Musculoskeletal Research Unit, School of Clinical Sciences, University of Bristol, Bristol BS10 5NB, UK. ${ }^{3}$ Lower Extremity and Gait Studies Program, School of Allied Health, La Trobe University, Bundoora, Victoria 3086, Australia.

Received: 26 September 2014 Accepted: 18 June 2015 Published online: 13 July 2015

\section{References}

1. Felson DT. Developments in the clinical understanding of osteoarthritis. Arthritis Res Ther. 2009;11:203.

2. Bijlsma JW, Berenbaum F, Lafeber FPJG. Osteoarthritis: an update with relevance for clinical practice. Lancet. 2011;377:2115-26.

3. Trivedi B, Marshall M, Belcher J, Roddy E. A systematic review of radiographic definitions of foot osteoarthritis in population-based studies. Osteoarthritis Cartilage. 2010;18:1027-35.

4. Thomas MJ, Roddy E, Zhang W, Menz HB, Hannan MT, Peat GM. The population prevalence of foot and ankle pain in middle and old age: a systematic review. Pain. 2011;152:2870-80.

5. Roddy E, Thomas MJ, Marshall M, Rathod T, Myers H, Menz HB, et al. The population prevalence of symptomatic radiographic foot osteoarthritis in community-dwelling older adults: cross-sectional findings from the Clinical Assessment Study of the Foot. Ann Rheum Dis. 2015;74:156-63.

6. Rathod T, Marshall M, Thomas MJ, Myers H, Menz HB, Thomas E, et al. Patterns of joint involvement in foot osteoarthritis: findings from the Clinical Assessment Study of the Foot [abstract]. Osteoarthritis Cartilage. 2014;22:S206.

7. Gorter K, de Poel S, de Melker R, Kuyvenhoven M. Variation in the diagnosis and management of common foot problems by GPs. Fam Pract. 2001;18:569-73.

8. Thomas MJ, Moore A, Roddy E, Peat G. "Somebody to say 'come on we can sort this'": a qualitative study of primary care consultation among older adults with symptomatic foot osteoarthritis. Arthritis Care Res. 2013;65:2051-5.

9. Lundgren P, Nester C, Liu A, Arndt A, Jones R, Stacoff A, et al. Invasive in vivo measurement of rear-, mid- and forefoot motion during walking. Gait Posture. 2008;28:93-100.
10. Ebalard M, Le Henaff G, Sigonney G, Lopes R, Kerhousse G, Brilhault J, et al. Risk of osteoarthritis secondary to partial or total arthrodesis of the subtalar and midtarsal joints after a minimum follow-up of 10 years. Orthop Traumatol Surg Res. 2014;100:S231-7.

11. Saltzman CL, Fehrle MJ, Cooper RR, Spencer EC, Ponseti IV. Triple arthrodesis: twenty-five and forty-four-year average follow-up of the same patients. J Bone Joint Surg Am. 1999;81:1391-402.

12. Roddy E, Myers H, Thomas MJ, Marshall M, D'Cruz D, Menz HB, et al. The clinical assessment study of the foot (CASF): study protocol for a prospective observational study of foot pain and foot osteoarthritis in the general population. J Foot Ankle Res. 2011;4:22.

13. Ware Jr J, Kosinski M, Keller SD. A 12-item Short-Form Health Survey: construction of scales and preliminary tests of reliability and validity. Med Care. 1996;34:220-33.

14. Zigmond AS, Snaith RP. The hospital anxiety and depression scale. Acta Psychiatr Scand. 1983;67:361-70.

15. Dufour AB, Broe KE, Nguyen US, Gagnon DR, Hillstrom HJ, Walker AH, et al. Foot pain: is current or past shoewear a factor? Arthritis Rheum. 2009;61:1352-8.

16. Garrow AP, Papageorgiou AC, Silman AJ, Thomas E, Jayson MI, Macfarlane GJ. Development and validation of a questionnaire to assess disabling foot pain. Pain. 2000;85:107-13.

17. Garrow AP, Silman AJ, Macfarlane GJ. The Cheshire Foot Pain and Disability Survey: a population survey assessing prevalence and associations. Pain. 2004; 110:378-84

18. Menz HB, Munteanu SE, Landorf KB, Zammit GV, Cicuttini FM. Radiographic classification of osteoarthritis in commonly affected joints of the foot. Osteoarthritis Cartilage. 2007;15:1333-8.

19. Chatterton BD, Muller S, Thomas MJ, Menz HB, Rome K, Roddy E. Inter and intra-rater repeatability of the scoring of foot pain drawings. J Foot Ankle Res. 2013;6:44.

20. Roddy E, Muller S, Thomas E. Defining disabling foot pain in older adults: further examination of the Manchester foot pain and disability index. Rheumatology (Oxford). 2009;48:992-6.

21. Carpenter JR, Kenward MG. Multiple imputation and its application. Chichester: Wiley; 2013.

22. Muller S, Roddy E. A rasch analysis of the Manchester foot pain and disability index. J Foot Ankle Res. 2009;2:29.

23. Rubin DB. Multiple imputation for nonresponse in surveys. New York: Wiley; 1987.

24. Lawrence JS. Osteo-arthrosis. In: Lawrence JS, editor. Rheumatism in populations. London: William Heinemann Medical Books; 1977. p. 98-155.

25. Marshall M, Peat G, Nicholls E, van der Windt D, Myers H, Dziedzic K. Subsets of symptomatic hand osteoarthritis in community-dwelling older adults in the United Kingdom: prevalence, inter-relationships, risk factor profiles and clinical characteristics at baseline and 3-years. Osteoarthritis Cartilage. 2013;21:1674-84.

26. Leng GC, Fowkes FGR. The Edinburgh Claudication Questionnaire: an improved version of the WHO/Rose Questionnaire for use in epidemiological surveys. J Clin Epidemiol. 1992;45:1101-9.

27. Papageorgiou AC, Croft PR, Ferry S, Jayson MI, Silman AJ. Estimating the prevalence of low back pain in the general population. Evidence from the South Manchester back pain survey. Spine. 1995;20:1889-94.

28. Birrell F, Croft P, Cooper C, Hosie G, Macfarlane GJ, Silman A. Radiographic change is common in new presenters in primary care with hip pain. PCR Hip Study Group. Rheumatology (Oxford). 2000;39:772-5.

29. Lacey RJ, Lewis M, Jordan K, Jinks C, Sim J. Interrater reliability of scoring of pain drawings in a self-report health survey. Spine. 2005;30:E455-8.

30. Urwin M, Symmons D, Allison T, Brammah T, Busby H, Roxby M, et al. Estimating the burden of musculoskeletal disorders in the community: the comparative prevalence of symptoms at different anatomical sites, and the relation to social deprivation. Ann Rheum Dis. 1998;57:649-55.

31. Kellgren JH, Moore R. Generalized osteoarthritis and Heberden's nodes. $\mathrm{Br}$ Med J. 1952;1:181-7.

32. Berenbaum F. Osteoarthritis as an inflammatory disease (osteoarthritis is not osteoarthrosis!). Osteoarthritis Cartilage. 2013;21:16-21.

33. Felson DT. Osteoarthritis as a disease of mechanics. Osteoarthritis Cartilage. 2013;21:10-5.

34. Brandt KD, Dieppe P, Radin E. Etiopathogenesis of osteoarthritis. Med Clin North Am. 2009;93:1-24.

35. Felson DT, Goggins J, Niu J, Zhang Y, Hunter DJ. The effect of body weight on progression of knee osteoarthritis is dependent on alignment. Arthritis Rheum. 2004;50:3904-9. 
36. Niu J, Zhang YQ, Torner J, Nevitt M, Lewis CE, Aliabadi P, et al. Is obesity a risk factor for progressive radiographic knee osteoarthritis? Arthritis Rheum 2009;61:329-35.

37. Menz HB, Munteanu SE, Zammit GV, Landorf KB. Foot structure and function in older people with radiographic osteoarthritis of the medial midfoot. Osteoarthritis Cartilage. 2010;18:317-22.

38. Rao S, Baumhauer JF, Nawoczenski DA. Is barefoot regional plantar loading related to self-reported foot pain in patients with midfoot osteoarthritis. Osteoarthritis Cartilage. 2011;19:1019-25.

39. Pearce CJ, Calder JD. Surgical anatomy of the midfoot. Knee Surg Sports Traumatol Arthrosc. 2010;18:581-6.

40. Butterworth PA, Landorf KB, Smith SE, Menz HB. The association between body mass index and musculoskeletal foot disorders: a systematic review. Obes Rev. 2012;13:630-42.

41. Aspden RM. Obesity punches above its weight in osteoarthritis. Nat Rev Rheumatol. 2011;7:65-8.

42. Patel A, Rao S, Nawoczenski D, Flemister AS, DiGiovanni B, Baumhauer JF. Midfoot arthritis. J Am Acad Orthop Surg. 2010;18:417-25.

43. Muthuri SG, McWilliams DF, Doherty M, Zhang W. History of knee injuries and knee osteoarthritis: a meta-analysis of observational studies. Osteoarthritis Cartilage. 2011;19:1286-93.

44. Goud A, Khurana B, Chiodo C, Weissman BN. Women's musculoskeletal foot conditions exacerbated by shoe wear: an imaging perspective. Am J Orthop (Belle Mead NJ). 2011;40:183-91.

45. Jinks $C$, Jordan K, Ong BN, Croft P. A brief screening tool for knee pain in primary care (KNEST). 2. Results from a survey in the general population aged 50 and over. Rheumatology (Oxford). 2004;43:55-61.

46. Dziedzic K, Thomas E, Hill S, Wilkie R, Peat G, Croft PR. The impact of musculoskeletal hand problems in older adults: findings from the North Staffordshire Osteoarthritis Project (NorStOP). Rheumatology (Oxford). 2007:46:963-7.

47. Menz HB, Jordan KP, Roddy E, Croft PR. Musculoskeletal foot problems in primary care: what influences older people to consult? Rheumatology (Oxford). 2010;49:2109-16.

48. National Institute for Health and Care Excellence. Osteoarthritis: care and management in adults. NICE clinical guideline. London: Royal College of Physicians; 2014.

49. Rao S, Baumhauer JF, Becica L, Nawoczenski DA. Shoe inserts alter plantar loading and function in patients with midfoot arthritis. J Orthop Sports Phys Ther. 2009;39:522-31.

50. Drakonaki EE, Kho JSB, Sharp RJ, Ostlere SJ. Efficacy of ultrasound-guided steroid injections for pain management of midfoot joint degenerative disease. Skeletal Radiol. 2011;40:1001-6.

51. Mann RA, Prieskorn D, Sobel M. Mid-tarsal and tarsometatarsal arthrodesis for primary degenerative osteoarthrosis or osteoarthrosis after trauma. J Bone Joint Surg Am. 1996;78:1376-85.

52. Wills AK, Black S, Cooper R, Coppack RJ, Hardy R, Martin KR, et al. Life course body mass index and risk of knee osteoarthritis at the age of 53 years: evidence from the 1946 British birth cohort study. Ann Rheum Dis. 2012:71:655-60.

\section{Submit your next manuscript to BioMed Central and take full advantage of:}

- Convenient online submission

- Thorough peer review

- No space constraints or color figure charges

- Immediate publication on acceptance

- Inclusion in PubMed, CAS, Scopus and Google Scholar

- Research which is freely available for redistribution 